\title{
Discussão sobre humanização do parto: atuação do enfermeiro na Atenção Primária à Saúde
}

\author{
Discussion on humanizing childbirth: nursing behaviors in Primary Health Care \\ Cuestión sobre la humanización del nacimiento: el papel de las enfermeras en la Atención \\ Primaria de Salud
}

Bruna Galvani Bertoldo ${ }^{1 *}$, Rossano Sartori Dal Molin².

\begin{abstract}
RESUMO
Objetivo: Identificar a atuação do enfermeiro no pré-natal e a existência da discussão entre cesárea e parto normal humanizado na Atenção Primária a Saúde. Métodos: Trata-se de um estudo do tipo revisão integrativa de literatura em que a coleta de dados foi realizada nas bases de dados Scientific Electronic Library Online (SCIELO), Base de Dados de Enfermagem (BDENF) e Literatura Latino-Americana e do Caribe em Ciências da Saúde (LILACS), sendo utilizado como descritores: parto humanizado, cesárea, cuidado pré-natal, atenção primária à saúde, enfermagem obstétrica. Foram incluídos artigos disponíveis em língua portuguesa, publicados nos últimos dez anos. Resultados: No total foram encontrados 589 artigos, sendo elencados nove artigos para análise. Apenas dois artigos indicaram a existência de orientações sobre os tipos de parto e a presença de discussão sobre cesárea e parto normal humanizados entre enfermeiro e paciente. Os demais estudos mostraram a inexistência de orientações e discussão sobre o tema causando fragilidade no atendimento e insegurança à gestante. Considerações finais: $A$ análise realizada mostrou que com pouca frequência os enfermeiros fornecem informações sobre o parto causando desamparo as gestantes e dificultando o protagonismo da mulher durante o parto.
\end{abstract}

Palavras-chave: Cuidado pré-natal, Enfermagem, Atenção primária à saúde, Parto humanizado.

\begin{abstract}
Objective: To identify the role of nurses in prenatal care and the existence of a discussion between cesarean section and humanized normal birth in Primary Health Care. Methods: This is an integrative literature review type study in which data collection was performed in the Scientific Electronic Library Online (SCIELO), Base de Dados de Enfermagem (BDENF) and Literatura Latino-Americana e do Caribe em Ciências da Saúde (LILACS), it was being used as descriptors: humanized delivery, cesarean section, prenatal care, primary health care, obstetric nursing. The articles included were avaliable in Portuguese and were published in the last ten years. Results: In total, 589 articles were found, with nine articles being listed for analysis. Only two articles indicated the existence of guidelines on the types of delivery and the presence of a discussion about humanized cesarean section and normal birth between nurse and patient. The other studies showed the lack of guidance and discussion on the subject, causing weakness in the care and insecurity for pregnant women. Final considerations: The analysis carried out showed that nurses rarely provide information about childbirth, causing the pregnant women to feel helpless and making it difficult for women to play a leading role during childbirth.
\end{abstract}

Keywords: Prenatal care, Nursing, Primary health care, Humanizing childbirth.

${ }^{1}$ Centro Universitário da Serra Gaúcha (FSG), Caxias do Sul - RS. *E-mail: brunagbertoldo@gmail.com

SUBMETIDO EM: 12/2021

| ACEITO EM: 12/2021

PUBLICADO EM: 2/2022 


\section{RESÚMEN}

Objetivo: Identificar el rol del enfermero en la atención prenatal y la existencia de la discusión entre cesárea y parto natural humanizado en Atención Primaria de Salud. Métodos: Se trata de un estudio de tipo revisión integradora de la literatura en el que se realizó recolección de datos en el Scientific Electronic Library Online (SCIELO), Base de Dados de Enfermagem (BDENF) y Literatura Latino-Americana e do Caribe em Ciências da Saúde (LILACS), siendo utilizados como descriptores: parto humanizado, cesárea, atención prenatal, atención primaria de salud, enfermería obstétrica. Se incluyeron artículos disponibles en portugués, publicados en los últimos diez años. Resultados: En total, se encontraron 589 artículos, con 9 artículos listados para análisis. Solo 2 artículos indicaron la existencia de guías sobre los tipos de parto y la presencia de una discusión sobre cesárea humanizada y parto natural entre enfermera y paciente. Los otros estudios evidenciaron la falta de orientación y discusión sobre el tema, provocando debilidad en la atención e inseguridad para las embarazadas. Consideraciones finales: El análisis realizado mostró que las enfermeras brindan con poca frecuencia información sobre el parto, provocando que las gestantes se sientan indefensas y dificultando que las mujeres lleven a cabo un papel protagónico durante el parto.

Palabras clave: Atención prenatal, Enfermería, Atención primaria de salud, Parto humanizado.

\section{INTRODUÇÃO}

A Atenção Primária a Saúde (APS) é o primeiro nível de assistência à saúde e é caracterizada por um conjunto de ações no âmbito coletivo e individual. A atenção a saúde das gestantes nas Unidades de Saúde da Família (USF) ocorre por meio da realização do pré-natal, que segundo orientações do Ministério da Saúde (MS), é necessário iniciá-lo a partir da descoberta da gravidez, sendo recomendado a realização de no mínimo seis consultas para uma gestação de risco habitual. O MS, a fim de garantir a atenção a saúde das parturientes e do recém-nascido, instituiu, por meio da Portaria/GM ํo 569, de 01/06/2000, o Programa de Humanização no Pré-Natal e Nascimento (PHPN), que se fundamenta nos preceitos de que a humanização da Assistência Obstétrica e Neonatal é condição primária para o adequado acompanhamento do parto e do puerpério (MINISTÉRIO DA SAÚDE, 2021; SECRETARIA ESTADUAL DA SAÚDE, 2018).

O parto normal ainda é visto como um processo doloroso e sofrido para a mulher, todavia é natural e apresenta inúmeros benefícios para o binômio mãe-filho. Por conseguinte, a cesariana é considerada um avanço da medicina que permitiu a redução do índice de morbimortalidade materno infantil, entretanto provoca alguns prejuízos a mulher no pós-parto. Ambos os partos têm suas vantagens e desvantagens, contudo a humanização deve existir independente do parto escolhido. Entende-se por humanização o respeito a individualidade, dores e desejos da mulher, permitindo a gestante ser protagonista em seu parto, tomando as decisões e escolhas que a deixem mais confortáveis naquele momento único de sua vida (MINISTÉRIO DA SAÚDE 2002; MASCARENHAS VA et al., 2019).

Levando em consideração as orientações fornecidas as gestantes em relação ao parto, existe uma fragilidade no atendimento e na assistência às gestantes o que classifica a adequação do pré-natal como intermediária em muitas unidades de atendimento. A enfermagem tem um papel importante na propagação de informações, uma vez que conta com uma ferramenta fundamental, a consulta de enfermagem, que permite o desenvolvimento de uma assistência humanizada e eficaz à gestante (GONÇALVES MF et al., 2017; RODRIGUES FR et al., 2018).

O presente estudo foi desenvolvido com o objetivo de identificar a atuação do enfermeiro no pré-natal e a existência da discussão entre cesárea e parto normal humanizado na APS; assim como, descrever os benefícios do parto normal humanizado para o binômio mãe-filho, evidenciar a importância da consulta de enfermagem e a atuação do enfermeiro no pré-natal, e também a relevância da discussão entre o profissional enfermeiro e a gestante acerca dos tipos de partos, elencando suas vantagens e desvantagens. Essa pesquisa justificou-se devido a importância do tema, com o intuito de ressaltar a influência do enfermeiro no desenvolvimento de um pré-natal adequado e eficaz as gestantes. 


\section{MÉTODOS}

Trata-se de um estudo do tipo revisão integrativa da literatura com caráter analítico, em que essa metodologia proporciona a síntese do conhecimento e a incorporação da aplicabilidade de resultados de estudos significativos na prática. Esse método permite a generalização sobre determinados assuntos por diversos pesquisadores, em diferentes lugares e momentos; desse modo, é possível manter os interessados atualizados e facilitar as modificações nas práticas cotidianas como uma consequência da pesquisa (ROMAN AR e FRIEDLANDER MR, 1998; SOUZA MT et al., 2010).

Para o processo de seleção dos artigos o estudo foi norteado pela pergunta: "no pré-natal, existe a discussão entre o profissional enfermeiro e a gestante sobre parto cesárea e parto normal humanizado?". A coleta de dados foi realizada nas bases de dados Scientific Electronic Library Online (SCIELO), Base de Dados de Enfermagem (BDENF) e Literatura Latino-Americana e do Caribe em Ciências da Saúde (LILACS), sendo utilizado como descritores: parto humanizado, cesárea, cuidado pré-natal, atenção primária à saúde, enfermagem obstétrica.

Para a seleção dos artigos foram utilizados como critérios de inclusão artigos originais e completos, disponíveis em língua portuguesa de forma online e gratuita, publicados no período de 2011 a 2021 , que abordassem sobre a atuação do enfermeiro na discussão sobre parto normal humanizado e cesárea no âmbito da APS. Como critérios de exclusão foi determinado artigos que não estivessem disponibilizados eletronicamente em texto completo e que não respondessem à questão norteadora.

A análise dos dados foi realizada por meio de uma leitura criteriosa e com o auxílio de um instrumento que possibilitou a análise da qualidade das informações e a integração em relação aos critérios de inclusão. Essa ferramenta contava com os seguintes itens: título, autor(es), ano de publicação, base de dados, objetivos, resultados e conclusão, o que permitiu a identificação de estudos adequados e relacionados com o tema estudado.

Por se tratar de uma revisão integrativa da literatura não houve necessidade de submissão à avaliação do Comitê de Ética em Pesquisa. Todavia, todos os preceitos éticos referentes à privacidade e sigilo, quando necessário, legitimidade de informações e apresentação de todos os autores devidamente referenciados foram respeitados a fim de desenvolver uma pesquisa séria e de qualidade.

\section{RESULTADOS}

Foram selecionadas três bases de dados para a realização da coleta de dados da pesquisa: SCIELO, BDENF e LILACS. Inicialmente, foi realizado a pesquisa na base de dados SCIELO com os seguintes descritores: cuidado pré-natal, atenção primária a saúde, enfermagem, parto humanizado e cesárea. Nessa pesquisa foi encontrado 193 artigos e então utilizou-se alguns critérios de inclusão como texto completo, artigos em língua portuguesa e que estivessem entre o período de publicação dos anos 2011 a 2021. Com isso, restaram 160 estudos, em que foi realizado a leitura de seus resumos em busca de quais respondiam à questão norteadora da pesquisa para selecionar apenas os estudos compatíveis com a pesquisa a ser realizada. Logo, respeitando todos os critérios de inclusão, foram selecionados da base de dados SCIELO 4 artigos.

Diante da pesquisa realizada na base de dados BDENF, os descritores utilizados foram os mesmos citados anteriormente sendo encontrado um total de 98 artigos. Ao utilizar os critérios de inclusão de texto completo, artigos em língua portuguesa e compreendidos entre o período de 2011 a 2021, restaram 75 artigos. Após a leitura dos resumos desses estudos foram selecionados 3 artigos os quais respondiam à questão norteadora da pesquisa e integravam os critérios de inclusão.

Na pesquisa realizada na base de dados LILACS, foram utilizados os mesmos descritores citados acima e encontrado um total de 298 artigos. Após utilizar os critérios de inclusão restaram 221 estudos, que foram analisados mediantes seus resumos e selecionado 2 artigos os quais respondiam à questão norteadora $e$ apresentavam os critérios de inclusão propostos. 
No total da pesquisa forma encontrados 589 artigos, sendo reprovados 580 estudos após a aplicação dos critérios de inclusão e exclusão. Desse modo, foram selecionados para a realização da pesquisa de revisão 09 artigos, conforme demonstrado na Figura 1.

Figura 1 - Fluxograma de seleção dos artigos para Revisão Integrativa.

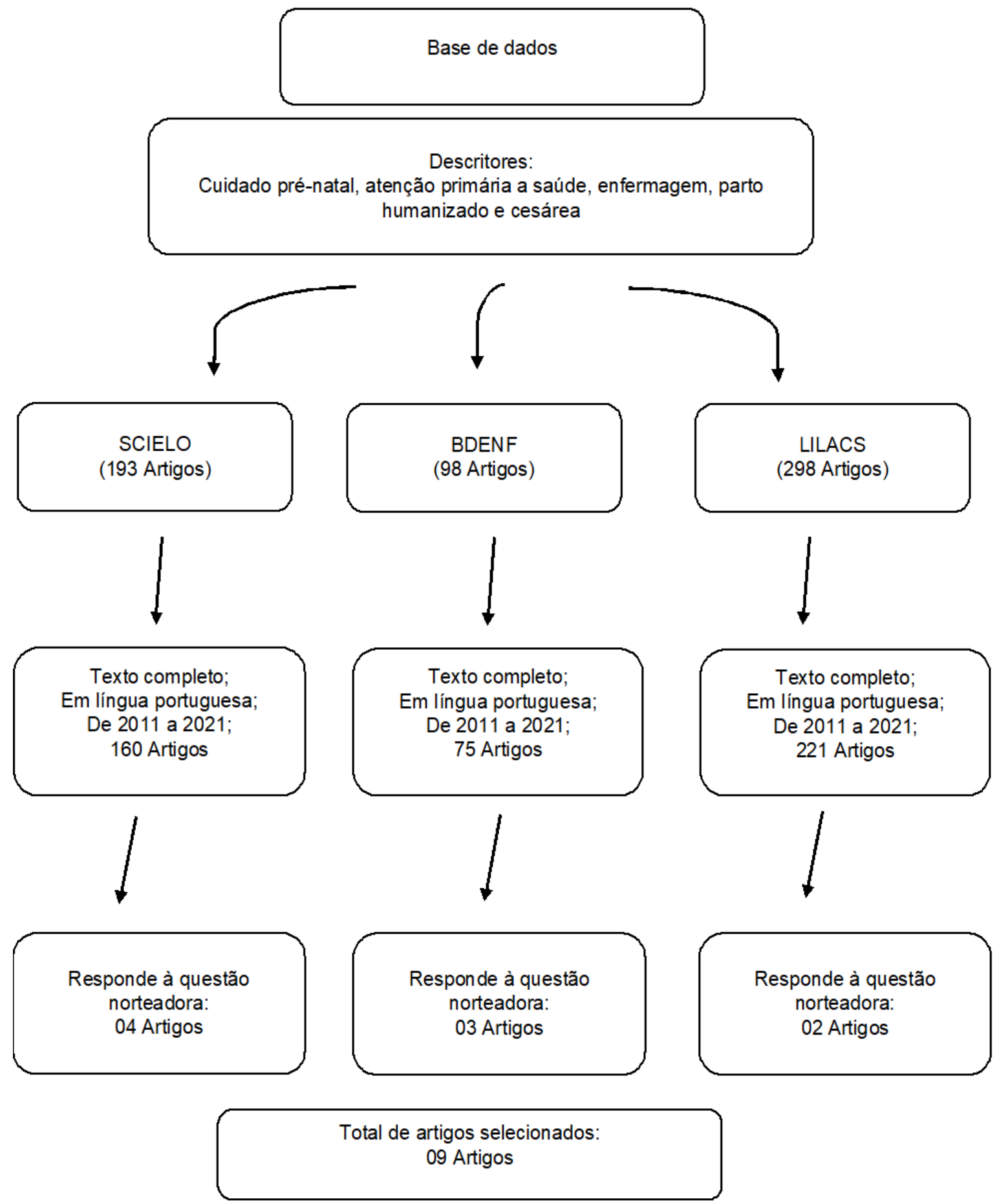

Fonte: Bertoldo BG, Molin RSD, 2021.

Para este estudo de revisão integrativa da literatura foram selecionados 9 artigos científicos que atenderam aos critérios de inclusão e exclusão estabelecidos. Os dados do Quadro 1 apresentam uma síntese com as informações mais relevantes dos estudos selecionados. 
Quadro 1- Apresentação da síntese das informações dos estudos selecionados para a revisão integrativa.

\begin{tabular}{|c|c|c|}
\hline & & \\
\hline Artigo, ano & Proposta & Principais achados \\
\hline Marques BL, et al., 2020. & $\begin{array}{l}\text { Expor os resultados obtidos mediante a pesquisa } \\
\text { realizada com puérperas sobre a adequação das } \\
\text { orientações em que receberam durante o pré- } \\
\text { natal, em relação ao profissional que emitiu } \\
\text { essas informações. }\end{array}$ & $\begin{array}{l}\text { Os resultados apontaram que a maioria das puérperas entrevistadas foram atendidas em } \\
\text { seu pré-natal, predominantemente, somente por médicos. } \\
\text { As orientações mais fornecidas às gestantes foram em relação a sinais de risco, risco de } \\
\text { automedicação e malefícios do tabagismo e álcool durante a gestação. } \\
\text { Em relação a adequação das orientações recebidas durante o pré-natal, a maioria } \\
\text { apresentou inadequação. Consultas realizadas por médico e enfermeiro, conjuntamente, } \\
\text { apresentaram maior chance de promover orientações adequadas às gestantes. }\end{array}$ \\
\hline Livramento DVP, et al., 2019. & $\begin{array}{l}\text { Apresentar a visão das gestantes frente ao } \\
\text { atendimento recebido durante o pré-natal no } \\
\text { âmbito da atenção primária a saúde. }\end{array}$ & $\begin{array}{l}\text { Segundos os resultados obtidos no estudo a maioria das gestantes entrevistadas } \\
\text { realizaram mais de seis consultas de pré-natal. Essas consultas, majoritariamente, foram } \\
\text { realizadas pelo profissional enfermeiro. } \\
\text { Em relação a percepção das gestantes frente ao atendimento recebido essas pacientes } \\
\text { manifestaram preferência pelo atendimento realizado pelo enfermeiro devido a percepção } \\
\text { mais humanizada desse profissional. Todavia, também relataram o descontentamento } \\
\text { com a rapidez de algumas consultas e ausência de informações verbais. } \\
\text { As gestantes manifestaram a ausência de informações sobre o parto, o que ocorreu } \\
\text { somente em alguns grupos de gestantes, entretanto as entrevistadas sentiram-se } \\
\text { despreparadas em relação a esse tema. }\end{array}$ \\
\hline Gonçalves MF, et al., 2017. & $\begin{array}{l}\text { Analisar a assistência prestada as gestantes } \\
\text { durante o pré-natal e a relação com as } \\
\text { orientações fornecidas referente ao parto na } \\
\text { atenção primária à saúde. }\end{array}$ & $\begin{array}{l}\text { Os resultados obtidos mostram que mais da metade das puérperas entrevistadas não } \\
\text { receberam qualquer tipo de orientação para o parto durante o pré-natal. Sendo que as } \\
\text { mulheres com cesariana prévia foram menos orientadas sobre o parto em relação as } \\
\text { mulheres com parto normal prévio. A pesquisa apresentou ineficiência da assistência } \\
\text { prestada as gestantes durante o pré-natal e ausência de continuidade no fornecimento } \\
\text { de informações sobre o parto ao longo do pré-natal. }\end{array}$ \\
\hline Rodrigues EM, et al., 2011. & $\begin{array}{l}\text { Analisar a compreensão dos enfermeiros frente a } \\
\text { utilização de protocolo de atribuições do } \\
\text { enfermeiro na assistência pré-natal, além de } \\
\text { identificar ações desenvolvidas e pontos que } \\
\text { facilitam e dificultam a inserção desse protocolo } \\
\text { na Estratégia de Saúde da Família. }\end{array}$ & $\begin{array}{l}\text { O estudo identificou em seus resultados que um grande número de enfermeiros } \\
\text { desconhecia as práticas realizadas em uma assistência pré-natal, uma vez que não } \\
\text { consideravam o atendimento do enfermeiro como parte do pré-natal, e sim, apenas o } \\
\text { atendimento médico. } \\
\text { Os resultados também mostraram que os enfermeiros acreditam que o protocolo na } \\
\text { assistência pré-natal ajuda a promover organização e otimizar o processo de trabalho. } \\
\text { Todavia, a maior dificuldade encontrada é a falta de capacitação teórico e práticas dos } \\
\text { profissionais para a utilização do protocolo na assistência às gestantes. }\end{array}$ \\
\hline Barbosa IS, et al., 2021. & $\begin{array}{l}\text { Apresentar a visão e o entendimento do } \\
\text { profissional enfermeiro em relação ao parto } \\
\text { humanizado. }\end{array}$ & $\begin{array}{l}\text { Os resultados encontrados mostram que os enfermeiros entrevistados entendem o parto } \\
\text { humanizado como aquele que respeita as escolhas e autonomia da mulher, sem a } \\
\text { utilização de práticas intervencionistas desnecessárias. } \\
\text { Os entrevistados também relatam que o enfermeiro pode atuar na escolha do parto da } \\
\text { mulher com informações, orientações, fornecimento de conhecimento sobre vantagens e } \\
\text { desvantagens sobre os tipos de parto a fim de promover conhecimento suficiente para as } \\
\text { gestantes escolherem a via de parto que desejam. }\end{array}$ \\
\hline
\end{tabular}

REAS | Vol.15(2) | DOI: https://doi.org/10.25248/REAS.e9460.2022

Página 5 de 11 


\begin{tabular}{|c|c|c|}
\hline Artigo, ano & Proposta & Principais achados \\
\hline Raznievski LFS, et al., 2020. & $\begin{array}{l}\text { Identificar a compreensão do profissional } \\
\text { enfermeiro da Atenção Básica sobre as boas } \\
\text { práticas de assistência ao parto em relação as } \\
\text { Práticas Baseadas em Evidências. }\end{array}$ & $\begin{array}{l}\text { Os resultados apresentados mostram que a maioria dos enfermeiros entrevistados } \\
\text { intercalam as consultas de pré-natal com o médico. } \\
\text { Também foi possível identificar que os entrevistados apresentavam uma compreensão } \\
\text { subjetiva, não baseada em evidências em relação a prestação de cuidados à mulher e ao } \\
\text { recém-nascido. } \\
\text { Os achados apresentam que mesmo não existindo instrumentos de apoio no serviço } \\
\text { estudado, as enfermeiras utilizam ferramentas e estratégias para promover orientações } \\
\text { adequadas em relação ao parto durante o pré-natal. }\end{array}$ \\
\hline Barros APZ, et al., 2017. & $\begin{array}{l}\text { Expor o entendimento dos enfermeiros que } \\
\text { realizam assistência pré-natal sobre o plano de } \\
\text { parto. }\end{array}$ & $\begin{array}{l}\text { Nos resultados apresentados foi possível identificar que os enfermeiros entrevistados } \\
\text { demonstraram desconhecimento sobre o documento Plano de Parto. } \\
\text { No local onde o estudo foi realizado o pré-natal era realizado, predominantemente, pelo } \\
\text { enfermeiro, que mesmo não conhecendo sobre o plano de parto realizava alguma de suas } \\
\text { etapas com as gestantes durante a assistência. } \\
\text { Os enfermeiros afirmaram que realizavam orientações sobre o parto às gestantes e seus } \\
\text { familiares. } \\
\text { É importante incentivar que os profissionais exerçam suas ações respeitando as diretrizes } \\
\text { do Ministério da Saúde e utilizem as ferramentas disponíveis, uma vez que nenhum } \\
\text { profissional conceituou corretamente o que é Plano de Parto. }\end{array}$ \\
\hline Chaves IS, et al., 2020. & $\begin{array}{l}\text { Identificar a satisfação das gestantes frente a } \\
\text { assistência recebida durante as consultas de } \\
\text { enfermagem no pré-natal. }\end{array}$ & $\begin{array}{l}\text { Os resultados apontam que as gestantes entrevistadas se sentiam confortáveis e mais } \\
\text { acolhidas nas consultas realizadas pelo enfermeiro. Também relatam que durante suas } \\
\text { consultas com o profissional enfermeiro suas dúvidas eram sanadas de forma tranquila e } \\
\text { que proporcionava conforto às gestantes. } \\
\text { Em relação a educação em saúde e orientações sobre o parto as gestantes relataram } \\
\text { que a maioria dessas informações eram transmitidas por meio de grupos de gestantes, } \\
\text { todavia a maioria das entrevistadas não participavam desses grupos. } \\
\text { Os resultados mostram que é importante o enfermeiro evitar o modelo de palestras para } \\
\text { fornecer orientações pertinentes durante o pré-natal, e sim abordar as mulheres sobre } \\
\text { fatores de risco, tipos de parto e métodos não farmacológicos para alívio da dor em } \\
\text { diálogos durante a consulta de enfermagem. }\end{array}$ \\
\hline Silva LA, et al., 2018. & $\begin{array}{l}\text { Apresentar a visão das gestantes em relação a } \\
\text { importância da assistência recebida no Programa } \\
\text { de Pré-natal durante o acompanhamento pré- } \\
\text { natal na Atenção Básica. }\end{array}$ & $\begin{array}{l}\text { Os resultados apontam uma insatisfação das gestantes no atendimento recebido pelo } \\
\text { médico com relatos de consultas rápidas, sem esclarecimento de dúvidas e também pela } \\
\text { não realização de procedimentos como verificar peso e medidas. } \\
\text { As entrevistadas relatam que o atendimento com o enfermeiro é muito mais humanizado } \\
\text { e que esses profissionais conversam e esclarecem suas dúvidas e preocupações. } \\
\text { Entretanto, o estudo mostra que é importante a realização de um acolhimento e escuta } \\
\text { ativa as mulheres a fim de promover segurança e assistência ampla às gestantes. }\end{array}$ \\
\hline
\end{tabular}

Fonte: Bertoldo BG, Molin RSD, 2021. Entretanto, o estudo mostra que é importante a realização de um acolhimento e
ativa as mulheres a fim de promover segurança e assistência ampla às gestantes. 


\section{DISCUSSÃO}

Por meio da análise dos estudos selecionados para essa revisão, foram desenvolvidas três categorias analíticas para melhor organização: atuação positiva do enfermeiro frente a discussão sobre os tipos de parto, ausência de informações pelo enfermeiro em relação aos tipos de parto e dificuldades para a realização de uma assistência qualificada no pré-natal.

\section{Atuação positiva do enfermeiro frente a discussão sobre os tipos de parto}

O acolhimento da gestante na atenção básica envolve a responsabilização pelo cuidado integral com início na recepção da usuária com escuta qualificada, avaliação da vulnerabilidade, criação de vínculo e atenção integral que envolvam a gestante e seus familiares. Segundo orientações do Ministério da Saúde é de competência do enfermeiro realizar a consulta de pré-natal de baixo risco, intercalando-as com o médico. Diante disso, o enfermeiro apresenta um papel fundamental no pré-natal das gestantes, umas vez que faz parte do seu trabalho orientar, acolher e promover um amplo cuidado a essas pacientes (MINISTÉRIO DA SAÚDE, 2013).

Barbosa IS, et al. (2021) realizou um estudo exploratório qualitativo em todas Unidades Básicas de Saúde do município de Paracuru-CE, com a participação de 10 enfermeiros a fim de compreender a percepção desses profissionais da APS acerca do parto humanizado. Com isso, foi possível identificar que os profissionais apresentavam ideias semelhantes quanto ao significado do parto humanizado, enfatizando o respeito, a autonomia e o protagonismo da mulher. Assim como, foi possível identificar que os enfermeiros entrevistados, durante suas consultas de enfermagem no pré-natal, realizavam orientações e dialogavam com as gestantes sobre os tipos de partos, vantagens e desvantagens a fim de promover informações e segurança para a paciente escolher o parto que desejava realizar.

Ao enfermeiro cabe a responsabilidade de promover um atendimento humanizado e esclarecedor, a fim de promover confiança à paciente e seus familiares. Com isso, esse profissional terá condições de desenvolver uma assistência de qualidade, promover orientações e escuta qualificada com o intuito de assegurar a gestante com informações relevantes sobre sua gestação e parto. Visto que, segundo recomendações do PHPN é por meio de um pré-natal de qualidade que a mulher terá participação como sujeito ativo durante todo o processo do trabalho de parto e parto (CORDEIRO EL, et al., 2018).

No estudo realizado por Barros APZ, et al., (2017) o pré-natal de risco habitual era realizado majoritariamente por enfermeiros da ESF, que mesmo demonstrando desconhecimento sobre o PP, apresentavam uma ideia de que o mesmo está relacionado aos direitos legais da gestante. Diante disso, os entrevistados declararam que durante suas consultas de enfermagem no pré-natal forneciam orientações e estabeleciam uma discussão com as gestantes acerca dos tipos de parto, benefícios do parto normal e vantagens e desvantagens dos partos normal e cesáreo.

O pré-natal é um momento de troca de experiências e promoção de orientações, além de promover às mulhres uma preparação física e psicológica para o parto e puerpério. Mesmo que em algumas situações o enfermeiro encontre dificuldades na assistência, é importante a realização de um cronograma de atividades, orientações e informações, assim como o desenvolvimento do PP que auxilia as mulheres no desenvolvimento de seu parto, promovendo conhecimento e segurança (CHAVES IS, et al, 2020).

Segundo o MS é direito das gestante receber assistência qualificada, atenção multiprofissional, além de escuta ativa da paciente e seus acompanhantes, informações sobre benefícios do parto fisiológico e elaboração de PP, ademais informações sobre seus direitos garantidos por lei em seu período gravídicopuerperal. Essas informações constam no Caderno de Atenção ao Pré-Natal de Baixo Risco proposto pelo MS e também é de responsabilidade do enfermeiro promover essas orientações e a ampla assistência as pacientes durante o período gravídico-puerperal (MINISTÉRIO DA SAÚDE, 2013).

\section{Ausência de informações pelo enfermeiro em relação aos tipos de parto}

A consulta de enfermagem é uma prática independente, realizada somente pelo enfermeiro, apresentando como objetivo proporcionar condições para a promoção da saúde das gestantes e melhorias na sua qualidade 
de vida. Conforme orientações do MS, é responsabilidade da equipe da APS promover assistência ampla e efetiva à gestante, para isso é necessário a identificação precoce das gestantes, acolhimento imediato e garantia de atendimento, acompanhamento periódico e contínuo, além de educação em saúde e orientações sobre aleitamento materno, tipos de parto e hábitos saudáveis de vida (MINISTÉRIO DA SAÚDE, 2013).

Diante da responsabilidade do enfermeiro frente a assistência pré-natal Raznievski LFS, et al. (2020) realizou um estudo sobre as percepções do enfermeiro em relação as boas práticas da assistência ao parto e nascimento. Essa pesquisa descritiva, exploratória com abordagem qualitativa foi desenvolvida com 20 enfermeiros de uma cidade da região central do Rio Grande do Sul, em que apontou que esses profissionais acreditavam que o pré-natal é um momento oportuno para fornecer orientações, todavia não recebem qualificações para fornecer orientações sobre assistência ao parto, trabalho de parto e parto.

No estudo proposto por Raznievski LFS, et al. (2020), encontra-se informações que é possível o entendimento que os enfermeiros, durante suas consultas de enfermagem realizam orientações às gestantes quanto a presença de acompanhante, práticas intervencionistas e as tranquilizam quanto a mitos que possam surgir durante a gestação. No entanto, orientações sobre o tipo de parto não são fornecidas às gestantes pelo enfermeiro, causando fragilidade na assistência, insegurança à paciente e desamparo.

O período do pré-natal é considerado o primeiro passo para um parto e nascimento humanizado, não obstante há necessidade de amparo, promoção de conhecimento, escuta qualificada, cuidado amplo e eficaz para que a gravidez possa ocorrer de forma tranquila e segura, com menos riscos de desfechos perinatais desfavoráveis (SEHNEM GD, et al., 2020; NEVES RG, et al., 2020).

O Sistema Único de Saúde (SUS) apresenta uma alta cobertura de pré-natal entre as gestantes usuárias, no entanto uma pesquisa realizada no ano de 2011/2012 analisou a adequação pré-natal conforme recomendações do MS e apontou que apenas $21,6 \%$ das mulheres receberam acompanhamento pré-natal apropriado. Ademais, estudos nacionais identificaram falhas dos profissionais de saúde em oferecer orientações sobre a gestação e parto às mulheres (TOMASI E, et al., 2017).

Marques BL, et al. (2020) realizou uma pesquisa com o intuito de analisar a associação entre adequação das orientações recebidas durante o pré-natal e o profissional que atendeu a gestante na APS. Para isso, por meio de um estudo quantitativo do tipo transversal realizado com uma amostra de 3.580 puérperas residentes em Santa Catarina, essa pesquisa pode identificar lacunas em relação as orientações ofertadas durante 0 acompanhamento pré-natal. Ao analisar os dados coletados com o estudo observou-se que a prevalência de adequação das orientações recebidas ficou em $18,6 \%$, sendo que as informações mais fornecidas as gestantes foram relacionadas aos sinais de risco, risco de automedicação e risco de fumo.

Identificou-se com a pesquisa acima que ainda existe uma hipervalorização de orientações de riscos em contraposição a orientações de autocuidado e autonomia. Uma vez que, durante a entrevista não houve relatos pelas puérperas de terem recebido orientações sobre o parto, seu direito de escolha de via de parto, ausência de práticas intervencionistas desnecessárias e seu protagonismo diante desse momento (MARQUES BL, et al., 2020).

Ainda no contexto relacionado a percepção das mulheres acerca do cuidado recebido durante o pré-natal, Livramento AVP, et al. (2019) por meio de um estudo qualitativo com 12 gestantes acompanhadas na APS do município de Florianópolis -SC, buscou compreender a concepção das gestantes frente à assistência recebida durante o pré-natal e apontou que, em sua maioria, as gestantes sentiram-se satisfeitas com o cuidado, todavia relataram a falta de orientações e esclarecimento de dúvidas, deixando-as confusas, sendo necessário buscar ajuda com familiares ou por meio de pesquisas na internet.

No estudo proposto por Livramento AVP, et al. (2019), inúmeros relatos foram relacionados a rapidez das consultas, incompreensão das orientações escritas e escassez de orientações verbais. Alguns relatos das gestantes mostram que as únicas informações recebidas ocorreram durante os grupos de gestantes, o que não substitui as orientações e assistência que deve ser realizada na consulta de enfermagem de modo íntimo e particular com cada gestante. 
O fornecimento de orientações e informações às gestantes e seus familiares não promove geração de custos ao SUS, sendo uma ação que dependentes, apenas, das atitudes dos profissionais. Segundo dados da Organização Mundial de Saúde (OMS) os índices de óbitos e doenças relacionadas à gravidez ainda estão em números elevados e são considerados como inaceitáveis, como mostra uma pesquisa realizada em 2015 em que apontou que cerca de 303 mil mulheres vieram a óbito por causas relacionadas à gravidez (OMS, 2021).

Gonçalves MF, et al., (2017) desenvolveu um estudo com o objetivo de avaliar a relação entre assistência pré-natal e orientações para o parto na APS. Trata-se de uma pesquisa transversal analítica realizada em uma maternidade pública da cidade de Londrina-PR, com uma população de 358 puérperas. Esse estudo mostrou a escassez de informações referentes ao parto ás gestantes, em que $52 \%$ das mulheres entrevistadas não receberam qualquer orientação para o parto durante seu acompanhamento pré-natal, sendo que destas, 38,2\% estavam gestando o primeiro filho. Toda gestação promove insegurança, medos e angustias as mulheres, tratando-se de primigestas todos esses sentimentos intensificam-se, por isso a ausência de informações para essas mulheres evidenciam a inadequação e ineficiência do cuidado prestado pelos enfermeiros e demais profissionais da saúde no pré-natal realizado na APS.

A fim de identificar práticas que possam auxiliar os enfermeiros em sua assistência durante o pré-natal, Rodrigues EM, et al. (2011) realizou um estudo qualitativo com enfermeiros de 15 Unidades Básicas de Saúde (UBS) da cidade de Divinópolis-MG, com o objetivo de conhecer a percepção dos enfermeiros diante do uso de protocolos e identificar ações de saúde, facilitadores e dificultadores do uso de protocolos. Com isso, a pesquisa apontou falhas na assistência causadas pelo desconhecimento do domínio da assistência pré-natal por parte dos enfermeiros, ausência de capacitação teórico e prática para uso dos protocolos e que mesmo havendo métodos que possam facilitar as consultas, alguns profissionais não a realizam e tampouco promovem orientações relacionadas a gestação e parto.

\section{Dificuldades para a realização de uma assistência qualificada no pré-natal}

O início precoce dos cuidados pré-natais e a assistência integral à gestante são condições importantes para realizar uma avaliação de condições que possam provocar vulnerabilidade à saúde da gestante e do neonato. $O$ enfermeiro é um dos profissionais fundamentais para efetuar o acolhimento pré-natal, por ser qualificado para promover ações com embasamento teórico e prático a fim de promover saúde, identificar anormalidades, prevenir doenças e promover um atendimento humanizado às gestantes e seus familiares (SEHNEM GD, et al. 2020).

Raznievski LFS, et al. (2020) em seu estudo sobre a percepção de enfermeiros na APS sobre boas práticas na assistência ao parto e Barbosa IS, et al. (2021) analisando a percepção dos enfermeiros sobre o parto humanizado fundamentam a importância do atendimento realizado por esses profissionais. Todavia, também apresentam relatos dos enfermeiros sobre as dificuldades encontradas em suas rotinas de trabalho, em que justificam as lacunas que ocorrem na assistência, em especial, na ausência de orientações pela grande demanda de gestantes, inadequação da estrutura física das unidades, dificuldade de adesão as gestantes aos grupos de apoio, além de falta de insumos como cartão de gestante, materiais para orientações e protocolos assistenciais que norteiem os profissionais.

Dados da pesquisa Nascer no Brasil evidenciaram que, aproximadamente, $89,6 \%$ das consultas pré-natais foram realizadas em unidades de APS o que corrobora a importância de uma assistência ampla e de qualidade às gestantes e aos seus familiares (VIELLAS EF, et al.,2014). É preciso trabalhar as equipes que atuam na APS para promover um acolhimento baseado no vínculo. Silva LA, et al. (2018) apresenta em seu estudo a insatisfação das gestantes frente ao seu atendimento nas Unidades Básicas, uma vez que era promovido um atendimento impositivo e pré-determinado e não baseado nas necessidades individuais de cada paciente.

Barros APZ, et al. (2017) e Rodrigues EM, et al. (2011) apresentam semelhanças nos resultados referente as dificuldades encontradas pelos enfermeiros para a realização de suas atividades. Ambos os estudos, ao serem analisados, mostram relatos desses profissionais sobre a ausência de capacitações, treinamentos e 
estratégias para auxiliar e melhorar o cuidado à gestante e aos seus familiares durante o pré-natal. Os enfermeiros justificam que o acolhimento dessas pacientes estão sendo prejudicados pelo desconhecimento e desatualização dos profissionais de saúde que muitas vezes recebem novos protocolos para serem utilizados na assistência, porém sem capacitações causando dúvidas para os profissionais e debilitando a atenção à gestante.

A existência de fatores que intrincam a assistência é uma realidade que precisa ser corrigida na APS com capacitações, trabalho em equipe, aumento de número de profissionais e criação de estratégias para qualificar o cuidado com as gestantes e seus familiares. Visto que, esses paciente não podem ficar desassistidos ou sem orientações que pertecem a seus direitos durante o pré-natal e é responsabilidade do enfermeiro, de sua equipe e dos demais profissionais da saúde atuantes na APS promover atenção ampla, eficaz e segura aos pacientes, em especial, às gestantes (BARROS APZ, et al., 2017).

\section{CONSIDERAÇÕES FINAIS}

O pré-natal é um período que envolve diversas dúvidas e mudanças na vida das gestantes e seus familiares, e também é um período que necessita de acompanhamento e atenção. Além dos cuidados e orientações principais que as gestantes recebem durante o seu pré-natal na APS, as informações sobre o parto são importantíssimas e é um direito da mulher conhecer os benefícios do parto normal e poder escolher o tipo de parto que deseja. Diante da análise dos estudos apresentados foi possível identificar que as orientações sobre o parto, informações sobre parto normal e parto cesáreo ocorrem com pouquíssima frequência, causando desamparo às gestantes. Sendo assim, é função do enfermeiro instruir e promover uma discussão construtiva com as gestantes acerca desse assunto para que a mulher possa construir seu conhecimento sobre o assunto, tomar decisões e tonar-se protagonista em seu parto.

\section{REFERÊNCIAS}

1. BARBOSA IS, et al. Percepção do enfermeiro da atenção primária acerca do parto humanizado. Revista Enfermagem em Foco, 2020; 11(6): 35-41.

2. BARROS APZ, et al. Conhecimento de enfermeiras sobre plano de parto. Revista de Enfermagem da UFSM, 2017; 7(1): 69-79

3. CHAVES IS, et al. Consulta de pré-natal de enfermagem: satisfação das gestantes. Revista Online de Pesquisa: Cuidado é Fundamental, 2020; 814-819.

4. CORDEIRO EL, et al. A humanização na assistência ao parto e ao nascimento. Revista de Enfermagem UFPE On Line, 2018; 12(8): 2154-62.

5. GONÇALVES MF, et al. Pré-natal: preparo para o parto na atenção primária à saúde no sul do Brasil. Revista Gaúcha de Enfermagem, 2017; 38(3): e2016-0063.

6. LIVRAMENTO DVP, et al. Percepção de gestantes acerca do cuidado pré-natal na atenção primária à saúde. Revista Gaúcha de Enfermagem, 2019; 40: e20180211.

7. MARQUES BL, et al. Orientações às gestantes no pré-natal: a importância do cuidado compartilhado na atenção primária em saúde. Revista Escola Anna Nery, 2021; 25(1): e20200098.

8. MASCARENHAS VHA, et al. Evidências científicas sobre métodos não farmacológicos para alívio a dor do parto. Revista Acta Paulista de Enfermagem, 2019; 32(3): 350-7.

9. MINISTÉRIO DA SAÚDE. Caderno de Atenção Básica: atenção ao pré-natal de baixo risco. Brasil, 2013. Disponível em: https://bvsms.saude.gov.br/bvs/publicacoes/cadernos_atencao_basica_32_prenatal.pdf. Acessado em: 05 de outubro de 2021.

10. MINISTÉRIO DA SAÚDE. Programa Humanização do Parto: Humanização no pré-natal e Nascimento. Brasil, 2002. Disponível em: https://bvsms.saude.gov.br/bvs/publicacoes/parto.pdf. Acessado em: 25 de agosto de 2021.

11. MINISTÉRIO DA SAÚDE. Secretaria de Atenção Primária à Saúde. Brasil, 2021. Disponível em: https://aps.saude.gov.br/smp/smpoquee. Acessado em: 25 de agosto de 2021.

12. NEVES GR, et al. Pré-natal no Brasil: estudo transversal do programa de melhoria do acesso e da qualidade da atenção básica, 2014. Revista Epidemiologia e Serviços de Saúde, 2020; 29(1):e2019019.

13. ORGANIZAÇÃO MUNDIAL DE SAÚDE (OMS). Organização Pan-Americana da Saúde. Disponível em: https://www.paho.org/pt/node/63100. Acessado em: 12 de outubro de 2021.

14. RAZNIEVSKI LFS, et al. Boas práticas de assistência ao parto e nascimento: percepção de enfermeiras da atenção 
básica. Revista de Enfermagem da UFSM, 2020; 34(10):1-18.

15. RODRIGUES EM, et al. Protocolo na assistência pré-natal: ações, facilitadores e dificuldades dos enfermeiros da Estratégia de Saúde da Família. Revista da Escola de Enfermagem da Universidade de São Paulo (USP), 2011; 45(5): 1041-1047.

16. RODRIGUES FR, et al. Pré-natal humanizado: estratégias de enfermagem na preparação para o parto ativo. Revista Saúde em Foco, 2018; 10:89-100.

17. ROMAN AR, FRIEDLANDER MR. Revisão integrativa aplicada à enfermagem. Revista Cogitare Enfermagem, 1998; 3(2): 109-112.

18. SECRETARIA ESTADUAL DA SAÚDE, Guia do Pré-Natal na Atenção Básica. Rio Grande do Sul, 2018. Disponível em: https://atencaobasica.saude.rs.gov.br/guia-do-pre-natal-na-atencao-basica. Acessado em: 25 de agosto de 2021.

19. SEHNEM GD, et al. Consulta de pré-natal na atenção primária à saúde: fragilidades e potencialidades da intervenção de enfermeiros brasileiros. Revista de Enfermagem Referência, 2020; 1: 2182-2883.

20. SILVA LA, et al. A humanização do cuidado pré-natal na perspectiva valorativa das mulheres gestantes. Revista Online de Pesquisa: Cuidado É Fundamental, 2018; 10(4): 1014-1019.

21. SOUZA MT, et al. Revisão integrativa: o que é e como fazer. Revista Einstein, 2010; 8(1):102-6.

22. TOMASI E, et al. Qualidade da atenção pré-natal na rede básica de saúde do Brasil: indicadores e desigualdades sociais. Revista Cadernos de Saúde Pública, 2017; 33(3): e00195815.

23. VIELLAS EF, et al. Assistência pré-natal no Brasil. Cadernos de Saúde Pública, 2014, 30(1): 85-100. 